PROCEEDINGS OF THE

AMERICAN MATHEMATICAL SOCIETY

Volume 131, Number 1, Pages 103-112

S 0002-9939(02)06556-5

Article electronically published on May 29, 2002

\title{
A NON-STANDARD PROOF OF THE BRIANÇON-SKODA THEOREM
}

\author{
HANS SCHOUTENS
}

(Communicated by Wolmer V. Vasconcelos)

\begin{abstract}
Using a tight closure argument in characteristic $p$ and then lifting the argument to characteristic zero with the aid of ultraproducts, I present an elementary proof of the Briançon-Skoda Theorem: for an $m$-generated ideal $\mathfrak{a}$ of $\mathbb{C}\left[\left[X_{1}, \ldots, X_{n}\right]\right]$, the $m$-th power of its integral closure is contained in $\mathfrak{a}$. It is well-known that as a corollary, one gets a solution to the following classical problem. Let $f$ be a convergent power series in $n$ variables over $\mathbb{C}$ which vanishes at the origin. Then $f^{n}$ lies in the ideal generated by the partial derivatives of $f$.
\end{abstract}

\section{INTRODUCTION}

The main aim of this paper is to provide an alternative proof of the following result. (We will write $\bar{I}$ for the integral closure of an ideal $I$.)

Theorem A (Briançon-Skoda). For every m-generated ideal $\mathfrak{a}$ in $\mathbb{C}\left[\left[X_{1}, \ldots, X_{n}\right]\right]$, we have an inclusion $\overline{\mathfrak{a}^{m}} \subset \mathfrak{a}$.

It is well-known that it has the following corollary 1

Theorem B. Let $f \in \mathbb{C}\left[\left[X_{1}, \ldots, X_{n}\right]\right]$ be a convergent power series without constant term. Let $\mathfrak{J}(f)$ denote the Jacobian ideal of $f$, that is to say, the ideal generated by all partial derivatives $\partial f / \partial X_{i}$. Then $f^{n} \in \mathfrak{J}(f)$.

Since the formal power series ring is faithfully flat over the ring of convergent power series, we may drop the requirement in Theorem B that $f$ is convergent and work with formal power series instead. With the right characterization of integral closure (Fact 2.2), it is an easy exercise on the chain rule to show that $f$ lies in the integral closure $\overline{\mathfrak{J}(f)}$ of $\mathfrak{J}(f)$ (Fact 5.1). Therefore, an application of Theorem A with $\mathfrak{a}=\mathfrak{J}(f)$ and $m=n$ proves Theorem $\mathbb{B}$ since always $(\overline{\mathfrak{a}})^{n} \subset \overline{\mathfrak{a}^{n}}$.

In fact, Theorem $\AA$ holds in much more generality, by work of Lipman, SathaYe and Teissier in [11] and 12, where the ring $A$ may be taken to be any regular or even pseudo-rational local ring and where one obtains an inclusion $\overline{\mathfrak{a}^{m+l}} \subset$ $\mathfrak{a}^{l+1}$, for all $l \in \mathbb{N}$. Below, I present an amazingly quick proof of Theorem A in positive characteristic, due to Hochster and HunEke (Fact 3.4 together with

Received by the editors April 6, 2001 and, in revised form, September 3, 2001.

2000 Mathematics Subject Classification. Primary 13A35, 13B22, 12L10.

Key words and phrases. Briançon-Skoda, tight closure, ultraproducts.

${ }^{1}$ According to WALL in [18, the question was originally posed by MATHER. It was first solved by BRIANÇON-SKODA in [5], using transcendental methods. 
Fact 3.3. I only explain the case $l=0$, but without effort the general case follows along the same lines). In fact, using their notion of tight closure in characteristic zero, the result extends to characteristic zero as well, thus providing an alternative proof of Theorem B. However, the very definition of tight closure in the ring $\mathbb{C}\left[\left[X_{1}, \ldots, X_{n}\right]\right]$ requires a substantial analysis of descent and genericity. Moreover, most of its properties can only be proven by means of a highly non-trivial Néron desingularization theorem of Artin-Rotthaus proven in [3]. The main contribution of this paper is to replace the use of this theory by a non-standard argument, explained in Section 4, together with the more elementary Artin Approximation 1, 2. for the ring of algebraic power series over $\mathbb{C}$. To wit, a system of equations over this ring is solvable if, and only if, it is solvable by means of formal power series. As a result, we have now a shorter proof of Theorem $\mathrm{A}$.

Most material in this paper is elementary and well-known; the only new ingredient is the approximate splitting part in Fact 4.3 relating the ultraproduct $A_{\infty}$ of the power series rings $\mathbb{F}_{p}^{\text {alg }}\left[\left[X_{1}, \ldots, X_{n}\right]\right]$ to the power series ring $\mathbb{C}\left[\left[X_{1}, \ldots, X_{n}\right]\right]$. The present proof relies on the Henselian property of the ultraproduct together with Artin Approximation. Originally, I intended to give a more elementary proof of this approximate splitting as well, but since it was merely a shortened version of ARTIN's original proof, I decided to publish this argument and the surrounding discussion in a separate paper. I did include a short argument for an improved version, where this time a true splitting over any finitely generated subalgebra is obtained (this is not used elsewhere in the paper; moreover, its proof relies on the less elementary Artin-Rotthaus Theorem). Ideally, we would like this splitting to hold in its strongest form, namely, that the ring $\mathbb{C}\left[\left[X_{1}, \ldots, X_{n}\right]\right]$ is a direct summand of the ultraproduct. At present, I only know this to be true for a single variable. It should be pointed out that Fact 4.3 in turn yields the Uniform Strong Artin Approximation of [4]; in fact the proof of Fact 4.3] is inspired by their ideas.

\section{Some final remarks.}

1.1. Remark. The term non-standard in the title refers to the use of ultraproducts.

1.2. Remark. Theorem $\mathrm{B}$ itself is false in characteristic $p$ (see Remark 5.2 below); nonetheless, its proof uses essentially characteristic $p$ methods.

1.3. Remark. The referee of an earlier version of this paper has pointed out to me-for which I thank him-that Theorem $[\mathrm{B}$ can be proven from the characteristic $p$ Briançon-Skoda Theorem (Fact 3.4 below) without any appeal to Artin Approximation or tight closure in characteristic zero. In fact, Theorem $\mathrm{A}$ itself admits such a reduction in the special case that $m$, the number of generators, is equal to $n$, the number of variables (which is the case needed to prove Theorem B). First, replace the ideal $\mathfrak{a}$ by $\mathfrak{a}+\left(X_{1}, \ldots, X_{n}\right)^{N}$ for some sufficiently high $N$, so that we may assume that $\mathfrak{a}$ is a primary ideal. Then replace $\mathfrak{a}$ by its minimal reduction, to reduce to an $n$-generated primary ideal. However, since $\mathfrak{a}$ is primary, it is the extension of a primary ideal in $\mathbb{C}\left[X_{1}, \ldots, X_{n}\right]_{\left(X_{1}, \ldots, X_{n}\right)}$ and by faithfully flat descent, we reduced the problem to the latter ring. In this case, reduction to characteristic $p$ can be carried out in a more elementary way, avoiding the use of Artin Approximation. In fact, analysis of the argument below shows that Theorem A admits an elementary proof for either $\mathbb{C}\left[X_{1}, \ldots, X_{n}\right]_{\left(X_{1}, \ldots, X_{n}\right)}$ or its Henselization, since in that case Fact 4.3 is immediate. 
I would also like to thank that referee for pointing out an erroneous approach for proving Fact 4.3

1.4. Remark. Although only stated here for $A=\mathbb{C}\left[\left[X_{1}, \ldots, X_{n}\right]\right]$, the present method easily proves the validity of Theorem $\mathrm{A}$ for any equicharacteristic regular local ring $A$. Indeed, after taking completion and making some base change of the ground field, we may assume by Cohen's Structure Theorem that $A=$ $K\left[\left[X_{1}, \ldots, X_{n}\right]\right]$, for $K$ an algebraically closed field of uncountable cardinality. Such a field $K$ is always an ultraproduct of fields of positive characteristic (this is the general version of Fact 4.2 below) so that the argument presented here easily extends to $K\left[\left[X_{1}, \ldots, X_{n}\right]\right]$.

\section{Integral ClOSURE}

In this section $A$ is an arbitrary Noetherian domain and $\mathfrak{a}$ is an ideal of $A$.

2.1. Definition. We say that $z$ lies in the integral closure of $\mathfrak{a}$, denoted $\overline{\mathfrak{a}}$, if there exist $a_{i} \in \mathfrak{a}^{i}$, such that

$$
z^{d}+a_{1} z^{d-1}+\cdots+a_{d}=0
$$

It follows immediately from the definition that $\overline{\mathfrak{a}}$ is contained in the radical of $\mathfrak{a}$. From the next characterization (see [19, Appendix 4] for proofs), it follows that $\overline{\mathfrak{a}}$ is an ideal.

2.2. Fact. The following are equivalent for an ideal $\mathfrak{a}$ of $A$ and an element $z \in A$ :

2.2.1. The element $z$ lies in the integral closure $\overline{\mathfrak{a}}$.

2.2.2. For each homomorphism $A \rightarrow V$ with $V$ a complete discrete valuation ring, the image of $z$ lies in $\mathfrak{a} V$.

2.2.3. There exists a non-zero $c \in A$, such that for all $m \in \mathbb{N}$, we have that $c z^{m} \in \mathfrak{a}^{m}$.

\section{Tight Closure}

Throughout this section, $A$ will denote a Noetherian domain with the extra assumption that its characteristic is $p>0$. Furthermore, $\mathfrak{a}=\left(f_{1}, \ldots, f_{m}\right)$ will be an arbitrary ideal of $A$. We will use $q$ to denote an arbitrary power of $p$. We will denote the Frobenius endomorphism $a \mapsto a^{q}$ on $A$ by $\mathbf{F r}_{q}$.

3.1. Fact. If $A$ is a power series ring over an algebraically closed field $K$, then $A$ is a finite free $\boldsymbol{F r}_{q}(A)$-module, where $\boldsymbol{F r}_{q}(A)$ is the image of $\boldsymbol{F r}_{q}$, that is to say, the subring of $A$ consisting of all $q$-th powers of elements in $A$.

Proof. In fact, Kunz proves that an arbitrary domain $A$ is regular if, and only if, the Frobenius $\mathbf{F r}_{p}$ is flat; for two quick proofs using either the BuchsBaum-EisEnBUD criterion or Cohen's Structure Theorem for complete regular equicharacteristic local rings, see [10, p. 12].

However, in the special case that $A=K\left[\left[X_{1}, \ldots, X_{n}\right]\right]$, the statement follows immediately, since $\operatorname{Fr}_{q}(A)=K\left[\left[X_{1}^{q}, \ldots, X_{n}^{q}\right]\right]$, so that the monomials $X^{\nu}$ with all entries of $\nu$ less than $q$, form a basis.

3.2. Definition. We will say that $z \in A$ belongs to the tight closure of $\mathfrak{a}$, denoted $\mathfrak{a}^{*}$, if there exists a non-zero $c \in A$, such that for all powers $q$ of $p$, we have

$$
c z^{q} \in\left(f_{1}^{q}, \ldots, f_{m}^{q}\right)\left(=\mathbf{F r}_{q}(\mathfrak{a}) A\right) .
$$


It is an easy exercise to check that $\mathfrak{a}^{*}$ is an ideal of $A$ containing $\mathfrak{a}$. From 2.2 .3 of Fact 2.2, it follows that $\mathfrak{a}^{*} \subset \overline{\mathfrak{a}}$.

3.3. Fact. If $A$ is a power series ring over an algebraically closed field $K$, then $\mathfrak{a}=\mathfrak{a}^{*}$, for every ideal $\mathfrak{a}$.

Proof. Again this holds in much more generality: every ideal in an arbitrary regular ring is equal to its own tight closure ([10, Theorem 1.3]). The proof uses the flatness of Frobenius in the following way. I will only present the proof in the case that interests us: $A$ is local with maximal ideal $\mathfrak{m}$ and $\mathbf{F r}_{q}$ is flat. Let $z \in \mathfrak{a}^{*}$, so that for each $q$, we have an equation (1), with $c$ non-zero and independent from $q$. Hence, for all $q$, we have that

$$
c \in\left(\mathbf{F r}_{q}(\mathfrak{a}) A: \mathbf{F r}_{q}(z)\right)=\operatorname{Fr}_{q}(\mathfrak{a}: z) A
$$

where we used the flatness of $\mathbf{F r}_{q}$ for the last equality. If $z \notin \mathfrak{a}$, then $(\mathfrak{a}: z) \subset \mathfrak{m}$. Therefore, by (2), $c \in \mathbf{F r}_{q}(\mathfrak{m}) A \subset \mathfrak{m}^{q}$, for all $q$, from which it follows that $c=0$, a contradiction.

The key result is now the following tight closure version of Theorem A.

3.4. Fact (Tight closure Briançon-Skoda). If $A$ is local ring (of positive characteristic) and $\mathfrak{a}$ an ideal in $A$ generated by $m$ elements, then $\overline{\mathfrak{a}^{m}} \subset \mathfrak{a}^{*}$.

Proof. Let $z \in \overline{\mathfrak{a}^{m}}$, where $\mathfrak{a}=\left(f_{1}, \ldots, f_{m}\right)$. By Condition 2.2.3 in Fact 2.2, it follows that there exists a non-zero $c \in A$, such that, $c z^{e} \in\left(\mathfrak{a}^{m}\right)^{e}$, for all $e \in \mathbb{N}$. Since $\mathfrak{a}$ is generated by the $m$ elements $f_{j}$, any element in $\mathfrak{a}^{e m}$ is divisible by some $f_{j}^{e}$. In other words, we have that $c z^{e} \in\left(f_{1}^{e}, \ldots, f_{m}^{e}\right)$, for all $e \in \mathbb{N}$. Taking for $e$ powers of $p$ shows that $z \in \mathfrak{a}^{*}$.

Putting both facts together, we get a proof of Theorem $\mathrm{A}$ (the Briançon-Skoda Theorem) in positive characteristic.

\section{Lefschetz Principle}

In this section, we assume some familiarity with the ultraproduct construction (see for instance [9, §9.5] or [7, §6.7]). Let $\mathcal{U}$ be some non-principal ultrafilter on an infinite index set $W$ (below $W$ will be the set of prime numbers or the set of natural numbers $\mathbb{N}$ ). For each $w \in W$, pick a ring $B_{w}$. We define the ultraproduct of the $B_{w}$ as the quotient of the direct product $\prod_{w} B_{w}$ modulo the equivalence relation in which two tuples $\left(a_{w} \mid w \in W\right)$ and $\left(b_{w} \mid w \in W\right)$ are identified, if there exists $D \in \mathcal{U}$, such that $a_{w}=b_{w}$ for all $w \in D$. If the ultrafilter $\mathcal{U}$ is clear from the context, we simply say that $a_{w}$ and $b_{w}$ are equal for almost all $w$. We denote the ultraproduct by

$$
\lim _{w \rightarrow \infty} B_{w} \quad \text { or simply by } \quad B_{\infty}
$$

(we omit reference to the ultrafilter $\mathcal{U}$ ). One checks that $B_{\infty}$ is again a ring. We will denote the image of the sequence $\left(a_{w} \mid w \in W\right)$ in $B_{\infty}$ by $\lim _{w \rightarrow \infty} a_{w}$.

4.1. Fact (Łos's Theorem). If $F \in \mathbb{Z}\left[T_{1}, \ldots, T_{n}\right]$ and $a_{i \infty}=\lim _{w \rightarrow \infty} a_{i w}$ with $a_{i w} \in B_{w}$, then $F\left(a_{1 \infty}, \ldots, a_{n \infty}\right)=0$ in $B_{\infty}$ if, and only if, $F\left(a_{1 w}, \ldots, a_{n w}\right)=0$ in $B_{w}$ for almost all $w$. 
Proof. Straightforward. In fact Łos's Theorem takes the following more general form: if a collection of structures $B_{w}$ in a fixed language $\mathcal{L}$ satisfy a sentence in that language, then so does their ultraproduct $B_{\infty}$ with respect to some non-principal ultrafilter $\mathcal{U}$ on the index set (see for instance [9, Theorem 9.5.1]).

From now on, $\mathcal{U}$ is a non-principal ultrafilter on the set of prime numbers and all ultraproducts will be taken with respect to this ultrafilter. We denote the algebraic closure of the $p$ element field by $\mathbb{F}_{p}^{\text {alg }}$.

4.2. Fact (Lefschetz Principle). There is a non-canonical isomorphism between the ultraproduct of the $\mathbb{F}_{p}^{a l g}$ and the field of complex numbers $\mathbb{C}$.

Proof. Write $\mathbb{F}_{\infty}$ for the ultraproduct $\lim _{p \rightarrow \infty} \mathbb{F}_{p}^{\text {alg. }}$. We can express in an equational way that a ring is a field and that this field is algebraically closed. Therefore, by Fact $4.1 \mathbb{F}_{\infty}$ is an algebraically closed field. Let $l$ be a rational prime. For all rational primes $p \neq l$, we have that $l$ is a unit in $\mathbb{F}_{p}^{\text {alg }}$. By Fact $4.1 l$ is a unit in $\mathbb{F}_{\infty}$. Therefore $\mathbb{F}_{\infty}$ has characteristic zero. It is a well-known fact that an ultraproduct of countably many countable sets has the cardinality of the continuum. The statement follows, since Steinitz' Theorem says that any two algebraically closed fields of characteristic zero of the same uncountable cardinality are isomorphic (this follows easily from the fact that they have the same transcendence degree over $\mathbb{Q}$; for a model theoretic proof using quantifier elimination, or, equivalently, Chevalley's Theorem, see [9, Corollary 4.5.7]).

Unfortunately, the relationship between an arbitrary sequence of rings and their ultrapower is more complicated than in the case of fields. For our purposes, we need a Lefschetz Principle for power series, and I will discuss this now. Let $X=$ $\left(X_{1}, \ldots, X_{n}\right)$ be a finite set of variables. For the remainder of this section, we set

$$
A_{\infty}:=\lim _{p \rightarrow \infty} \mathbb{F}_{p}^{\text {alg }}[[X]]
$$

From Fact 4.2 it is clear that $\mathbb{C}$ embeds in $A_{\infty}$. Moreover, the $\lim _{p \rightarrow \infty} X_{i}$ are algebraically independent over $K_{\infty}$, so that we may view them again as variables $X_{i}$. This makes $\mathbb{C}[X]$ into a subring of $A_{\infty}$. Since being local or Henselian can be expressed equationally, it follows from Łos's Theorem (Fact 4.1) that $A_{\infty}$ is a local Henselian ring. Being Noetherian, however, does not carry over, and consequently $A_{\infty}$ is not Noetherian and neither is it separated in the $(X)$-adic topology. Although we will not need it here, one can also check that every Cauchy sequence in $A_{\infty}$ has a limit. However, it is not immediately obvious how to embed $\mathbb{C}[[X]]$ in $A_{\infty}$, since limits are no longer unique. Using the Artin-Rotthaus Theorem, one can show that such an embedding exists for each finitely generated subalgebra; see Theorem 4.5 below. However, to prove Theorem $\mathrm{A}$, we only need the following weaker version. Its first part is well-known and easy (see 4, Lemma 3.4]) and I briefly recall the argument; the second part requires some form of Artin Approximation over $\mathbb{C}^{2}$

4.3. Fact (Partial Lefschetz Principle for power series). If

$$
\mathfrak{i}:=\bigcap_{d}\left(X_{1}, \ldots, X_{n}\right)^{d} A_{\infty}
$$

\footnotetext{
${ }^{2}$ I opted in this paper to just quote ARTiN's result. Nonetheless, with some surgery of his argument, one can obtain a more elementary and direct proof. I intend to return to this and related issues in a future paper on model theoretic consequences of Artin Approximation.
} 
denotes the so-called ideal of infinitesimals at the origin, then we have an exact sequence

$$
0 \rightarrow \mathfrak{i} \rightarrow A_{\infty} \stackrel{\pi}{\longrightarrow} \mathbb{C}[[X]] \rightarrow 0
$$

Moreover, if there is a commutative diagram

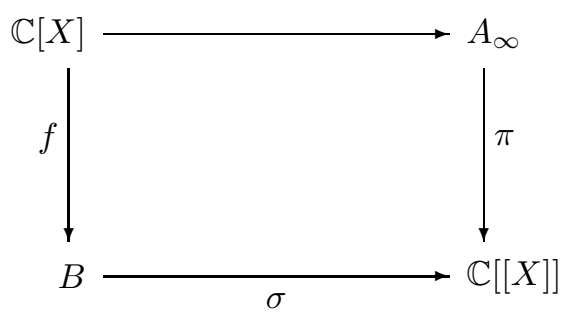

of $\mathbb{C}[X]$-algebra homomorphisms, with $B$ finitely generated over $\mathbb{C}[X]$, then for each $c \in \mathbb{N}$, we can find a $\mathbb{C}[X]$-algebra homomorphism $\delta_{c}: B \rightarrow A_{\infty}$, such that $\pi \delta_{c} \equiv \sigma$ $\bmod \left(X_{1}, \ldots, X_{n}\right)^{c}$.

Proof. Fix once and for all an isomorphism as in Fact 4.2. We start by defining $\pi$. Let $f_{\infty} \in A_{\infty}$. Choose $f_{p} \in A_{p}$, such that $\lim _{p \rightarrow \infty} f_{p}=f_{\infty}$ in $A_{\infty}$. Write each $f_{p}$ as $\sum a_{\nu p} X^{\nu}$, with $a_{\nu p} \in \mathbb{F}_{p}^{\text {alg }}$. Write $a_{\nu \infty} \in \mathbb{C}$ for $\lim _{p \rightarrow \infty} a_{\nu p}$ (after applying the isomorphism from Fact 4.2). Define $\pi\left(f_{\infty}\right)$ to be the power series $\sum a_{\nu \infty} X^{\nu}$. It follows from Fact 4.1 that $\pi$ is a $\mathbb{C}[X]$-algebra homomorphism and surjectivity is also clear. So it remains to show that $\mathfrak{i}$ is the kernel of $\pi$. If $f_{\infty} \in \mathfrak{i}$, then by Fact 4.1, for each $d$, there is $I_{d} \in \mathcal{U}$, such that for all $p \in I_{d}$, we have that $f_{p} \in\left(X_{1}, \ldots, X_{n}\right)^{d} \mathbb{F}_{p}^{a l g}[[X]]$. In particular, for each multi-index $\nu=\left(\nu_{1}, \ldots, \nu_{n}\right)$, we have that $a_{\nu p}=0$, for all $p \in I_{\nu_{1}+\cdots+\nu_{n}+1}$. It follows that $a_{\nu \infty}=0$. Since this holds for all $\nu$, we see that $\pi\left(f_{\infty}\right)=0$, as required. The converse holds by virtually the same argument.

To prove the last assertion, fix some $c \in \mathbb{N}$ and write $B$ as $\mathbb{C}[X, Y] /\left(P_{1}, \ldots, P_{s}\right)$, where $Y=\left(Y_{1}, \ldots, Y_{m}\right)$ are some extra variables. Let $\mathbf{y}$ be the image of $Y$ under the section $\sigma$, so that $P_{1}(\mathbf{y})=\cdots=P_{s}(\mathbf{y})=0$. Using Artin Approximation in characteristic zero 3 we can find a tuple $\mathbf{z}$ in the ring of algebraic power series $\mathbb{C}[[X]]^{\text {alg }}$, such that $P_{1}(\mathbf{z})=\cdots=P_{s}(\mathbf{z})=0$ and $\mathbf{y} \equiv \mathbf{z} \bmod \left(X_{1}, \ldots, X_{n}\right)^{c}$. Since $A_{\infty}$ is Henselian and since $\mathbb{C}[[X]]^{\text {alg }}$ is the Henselization of $\mathbb{C}[X]$ at the maximal ideal $\left(X_{1}, \ldots, X_{n}\right)$, it follows from the universal property of Henselizations that there is a (unique) $\mathbb{C}[X]$-algebra homomorphism $\lambda: \mathbb{C}[[X]]^{\text {alg }} \rightarrow A_{\infty}$. Uniqueness guarantees that $\pi \lambda$ is the identity on $\mathbb{C}[[X]]^{\text {alg }}$. Therefore, if we define $\delta_{c}$ to be the $\mathbb{C}[X]$-algebra homomorphism given by $Y \mapsto \lambda(\mathbf{z})$, we obtained the required factorization of $\sigma$.

4.4. Remark. In the absence of Quantifier Elimination and because $\pi$ is not an embedding, only positive existential sentences carry through from the power series rings $\mathbb{F}_{p}^{\text {alg }}[[X]]$ to the power series ring $\mathbb{C}[[X]]$. Therefore, it would be more appropriate to call Fact 4.3 an approximate $\exists^{+}$-Lefschetz Principle.

\footnotetext{
${ }^{3}$ One can for instance use the reasonably elementary argument of ARTIN's first paper [1] on the subject; in it he proves the result from formal to convergent power series, but the proof carries over without any problem to show the approximation property from formal to algebraic power series.
} 
Although not needed in this paper, it is of interest to have a strengthened version of Fact 4.3, albeit at the expense of using the highly non-trivial Artin-Rotthaus Theorem.

4.5. Theorem (Partial Lefschetz Principle). Suppose we have a commutative diagram

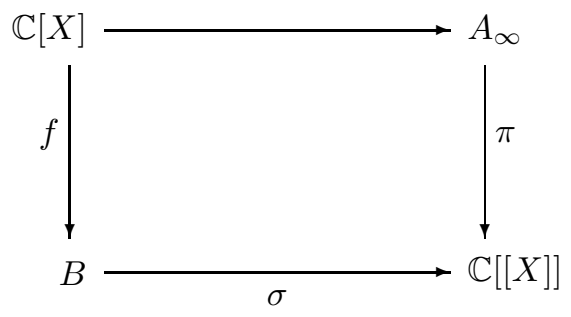

of $\mathbb{C}[X]$-algebra homomorphisms, with $B$ finitely generated over $\mathbb{C}[X]$. Then we can find a $\mathbb{C}[X]$-algebra homomorphism $\delta: B \rightarrow A_{\infty}$, such that $\pi \delta=\sigma$.

Proof. By [3. Corollary 5], given a diagram (3), one can write $\sigma$ as a composition of $\mathbb{C}[X]$-algebra homomorphisms $B \rightarrow \mathbb{C}[[X, Y]]^{\text {alg }}$ and $\tau: \mathbb{C}[[X, Y]]^{\text {alg }} \rightarrow \mathbb{C}[[X]]$, for some choice of variables $Y=\left(Y_{1}, \ldots, Y_{m}\right)$. To define $\delta: B \rightarrow A_{\infty}$, choose any lifting $\mathbf{z}$ in $A_{\infty}$ of $\tau(Y)$. Mapping $Y$ to $\mathbf{z}$ yields a $\mathbb{C}[X]$-algebra homomorphism $\mathbb{C}[X, Y] \rightarrow A_{\infty}$. Since $A_{\infty}$ is Henselian, this homomorphism admits a unique extension $\varepsilon: \mathbb{C}[[X, Y]]]^{\text {alg }} \rightarrow A_{\infty}$. Uniqueness guarantees that $\pi \varepsilon=\tau$. Restricting $\varepsilon$ to $B$ then yields the required homomorphism $\delta: B \rightarrow A_{\infty}$.

Similar Lefschetz Principles have been shown in 6, 17, 14, with substantially more difficult proofs. Nonetheless, I only know a positive answer to the following question when $n=1$.

4.6. Question (Lefschetz Principle). Is $\pi$ a split epimorphism, that is to say, is there a $\mathbb{C}[X]$-algebra homomorphism $\varepsilon: \mathbb{C}[[X]] \rightarrow A_{\infty}$, such that $\pi \varepsilon$ is the identity? Note that $\pi$ would then play a role analogous to the standard part operator from nonstandard analysis.

\section{Proofs of Theorems $\mathrm{A}$ and $\mathrm{B}$}

In this section, $A=\mathbb{C}[[X]]$, with $X=\left(X_{1}, \ldots, X_{n}\right)$. We fix some $f$ without constant term and define its Jacobian ideal $\mathfrak{J}(f)$ to be the ideal generated by the partial derivatives $\partial f / \partial X_{i}$. The following observation is classical.

5.1. Fact. With notation from above, $f$ lies in the integral closure $\overline{\mathfrak{J}(f)}$.

Proof. Using Fact 2.2, we have to show that $f \in \mathfrak{J}(f) V$, for every $A$-algebra $V$ which is a complete discrete valuation ring. By Cohen's Structure Theorem for complete equicharacteristic regular local rings (see for instance [13, (28.J) Corollary 2]), $V$ is of the form $K[[t]]$, with $t$ a single variable and $K$ an extension of $\mathbb{C}$. Let $x_{i}(t)$ denote the image of $X_{i}$ in $V$. The image of $f$ in $V$ is the power series $f(x(t))$. Factoring out the highest common power of $t$, we can write $f\left(x(t)=t^{e} u(t)\right.$, with $u(0) \neq 0$. From this last representation, it follows that

$$
\frac{d}{d t}(f(x(t)))=e t^{e-1} u+t^{e} \frac{d u}{d t}
$$


has $t$-order $e-1$. By the multi-variable chain rule on the other hand, we have that

$$
\frac{d}{d t}(f(x(t)))=\left.\frac{\partial f}{\partial X_{1}}\right|_{x(t)} \frac{d x_{1}}{d t}+\cdots+\left.\frac{\partial f}{\partial X_{n}}\right|_{x(t)} \frac{d x_{n}}{d t} .
$$

Comparing these two expressions, it follows that $t^{e-1} \in \mathfrak{J}(f) V$. Therefore $\mathfrak{J}(f) V$ also contains $f=t^{e} u$, as required.

5.2. Remark. Note that this fact is false in characteristic $p$ : in the above argument, $e$ could be divisible by $p$, so that $d f(x) / d t$ has order strictly bigger than $e-1$. For instance, the power series $f=(X-Y)^{2}+X^{p}$ provides a counterexample to both Fact 5.1 and Theorem B

By Fact 5.1 , Theorem $B$ now follows immediately from Theorem $\mathrm{A}$ for the ring $\mathbb{C}[[X]]$, since $\mathfrak{J}(f)$ is generated by $n$ elements. So it remains to prove Theorem $\mathrm{A}$

5.3. Proof of Theorem $\mathbf{A}$, Let $\mathfrak{a}=\left(f_{1}, \ldots, f_{m}\right)$ with $f_{i} \in \mathbb{C}[[X]]$ and let $f_{0} \in \overline{\mathfrak{a}^{m}}$. We need to show that $f_{0} \in \mathfrak{a}$. By assumption, there exist $g_{i} \in \mathfrak{a}^{m i}$, such that

$$
f_{0}^{d}+g_{1} f_{0}^{d-1}+\cdots+g_{d}=0 .
$$

In order to obtain similar integral equations in positive characteristic, we will use Fact $\left[4.3\right.$ in the following way. Let $A_{p}=\mathbb{F}_{p}^{\text {alg }}[[X]]$ and let $B$ be the $\mathbb{C}[X]$-subalgebra of $\mathbb{C}[[X]]$ generated by the $f_{i}$ and the $g_{i}$. Fix $c \in \mathbb{N}$. By Fact 4.3 , there exists a $\mathbb{C}[X]$-algebra homomorphism $\delta: B \rightarrow A_{\infty}$ such that $\pi \delta$ is congruent to the identity modulo $\left(X_{1}, \ldots, X_{n}\right)^{c}$. Choose $f_{i p}, g_{i p} \in A_{p}$, such that $\lim _{p \rightarrow \infty} f_{i p}=\delta\left(f_{i}\right)$ and $\lim _{p \rightarrow \infty} g_{i p}=\delta\left(g_{i}\right)$. Let $\mathfrak{a}_{p}$ be the ideal $\left(f_{1 p}, \ldots, f_{m p}\right)$ in $A_{p}$. It follows from tos's Theorem (Fact 4.1) and equation (5), after applying $\delta$ to it, that

$$
\left(f_{0 p}\right)^{d}+g_{1 p}\left(f_{0 p}\right)^{d-1}+\cdots+g_{d p}=0
$$

and that $g_{i p} \in\left(\mathfrak{a}_{p}\right)^{m i}$, for almost all $p$. Therefore, $f_{0 p}$ lies in the integral closure of $\left(\mathfrak{a}_{p}\right)^{m}$, for those $p$. By Fact 3.4, it follows that $f_{0 p}$ lies in the tight closure of $\mathfrak{a}_{p}$. By Fact 3.3 $\mathfrak{a}_{p}$ is equal to its own tight closure. Therefore, we can find $r_{i p} \in A_{p}$, such that

$$
f_{0 p}=r_{1 p} f_{1 p}+\cdots+r_{m p} f_{m p}
$$

for almost all $p$. By Fact 4.1 once more, we have a similar relation in $A_{\infty}$. More precisely, if $r_{i \infty}=\lim _{p \rightarrow \infty} r_{i p}$, then

$$
\delta\left(f_{0}\right)=r_{1 \infty} \delta\left(f_{1}\right)+\cdots+r_{m \infty} \delta\left(f_{m}\right) .
$$

Applying $\pi$ to this equation yields

$$
f_{0} \equiv \pi\left(r_{1 \infty}\right) f_{1}+\cdots+\pi\left(r_{m \infty}\right) f_{m} \quad \bmod \left(X_{1}, \ldots, X_{n}\right)^{c},
$$

showing that $f_{0} \in \mathfrak{a}+\left(X_{1}, \ldots, X_{n}\right)^{c}$. Since this holds for all $c$, we get by Krull's Intersection Theorem that $f_{0} \in \mathfrak{a}$, as required. 


\section{Tight closure proofs.}

5.3.1. Characteristic zero tight closure. The proof of HochsteR-HunEKE of Theorem A uses tight closure in characteristic zero. This is a closure operation on ideals in certain domains containing a field of characteristic zero (including complete local domains such as $\mathbb{C}[[X]])$. This closure operation then has the necessary formal properties to carry out the proof of Theorem A. To wit, any ideal in a regular local ring is equal to its own tight closure-the characteristic zero equivalent of Fact 3.3and, the tight closure Briançon-Skoda Theorem-the characteristic zero equivalent of Fact 3.4. However, to define this closure operation, one has to descend the relevant data to a finitely generated $\mathbb{Z}$-algebra and then take reduction modulo $p$. To return to the original ring, Artin Approximation 2] is needed, or, more accurately, the improvement by Artin-Rotthaus in [3]; see [10, Appendix 1] or [8] for more details.

5.3.2. Non-standard tight closure. The proof in this paper was inspired by a variant of tight closure in characteristic zero, which I termed non-standard tight closure in 16]. In that paper, some of the basic properties of non-standard tight closure are proven in the affine case, including the analogues of Facts 3.3 and 3.4. Provided the definition of non-standard tight closure as well as its properties can be extended to the case of power series rings-and this basically requires a Lefschetz Principle of the type proven in Theorem 4.5-, one would obtain yet another proof of Theorem $\mathrm{A}$ In the present article, however, I have opted to suppress any direct reference to this non-standard tight closure, since everything happens 'downstairs', that is to say, in characteristic $p$. A similar approach was taken in [15].

\section{REFERENCES}

1. M. Artin, On the solutions of analytic equations, Invent. Math. 5 (1968), 177-291. MR 38:344

2. - Algebraic approximation of structures over complete local rings, Inst. Hautes Études Sci. Publ. Math. 36 (1969), 23-58. MR 42:3087

3. M. Artin and C. Rotthaus, A structure theorem for power series rings, Algebraic Geometry and Commutative Algebra: in honor of M. Nagata (Tokyo), vol. I, Kinokuniya, 1988, pp. 3544. MR 90b:14006

4. J. Becker, J. Denef, L. van den Dries, and L. Lipshitz, Ultraproducts and approximation in local rings I, Invent. Math. 51 (1979), 189-203. MR 80k:14009

5. J. Briançon and H. Skoda, Sur la clôture intégrale d'un idéal de germes de fonctions holomorphes en un point de $C^{n}$, C. R. Acad. Sci. Paris 278 (1974), 949-951. MR 49:5394

6. J. Denef and H. Schoutens, On the decidability of the existential theory of $\mathbb{F}_{p}[[t]]$, Valuation Theory and its Applications (F.-V. Kuhlmann, S. Kuhlmann, and M. Marshall, eds.), Fields Institute Communications Series, Amer. Math. Soc., to appear, preprint on URL address

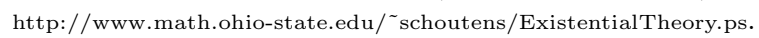

7. M. Fried and M. Jarden, Field arithmetic, Springer-Verlag, 1986. MR 89b:12010

8. M. Hochster and C. Huneke, Tight closure in equal characteristic zero, preprint available on URL address http://www.math.lsa.umich.edu/ ${ }^{\sim}$ hochster/tcz.ps.Z, 2000.

9. W. Hodges, Model theory, Cambridge University Press, Cambridge, 1993. MR 94e:03002

10. C. Huneke, Tight closure and its applications, Conference Board in Math. Sciences, vol. 88, Amer. Math. Soc., 1996. MR 96m:13001

11. J. Lipman and A. Sathaye, Jacobian ideals and a theorem of Briançon-Skoda, Michigan Math. J. 28 (1981), 199-222. MR 83m:13001

12. J. Lipman and B. Teissier, Pseudo-rational local rings and a theorem of Briançon-Skoda about integral closures of ideals, Michigan Math. J. 28 (1981), 97-116. MR 82f:14004

13. H. Matsumura, Commutative algebra, W.A. Benjamin, 1970. MR 42:1813 second ed. MR 82i: 13003 
14. H. Schoutens, Finitistic power series, preprint available on URL address http://www.math.ohiostate.edu/ schoutens/, 2001.

15. _ Lefschetz principle applied to symbolic powers, preprint available on URL address http://www.math.ohio-state.edu/ schoutens/SymbolicPowersLefschetz.ps, 2001.

16. _ Non-standard tight closure, preprint on URL address http://www.math.ohio-state.edu/ sschoutens/NonStandardTightClosure.ps, 2001.

17. $ـ$ Reduction modulo $p$ of power series with integer coefficients, preprint available on URL address http://www.math.ohio-state.edu/ schoutens/IntegerPowerSeries.ps, 2001.

18. C. Wall, Lectures on $C^{\infty}$ stability and classification, Proceedings of Liverpool SingularitiesSymposium I, Lect. Notes in Math., vol. 192, Springer-Verlag, 1971, pp. 178-206. MR 44:2244

19. O. Zariski and P. Samuel, Commutative algebra, Van Nostrand, Princeton, 1960. MR 22:11006

Department of Mathematics, 100 Math Tower, Ohio State University, Columbus, Оніо 43210

E-mail address: schoutens@math.ohio-state.edu

URL: http://www.math.ohio-state.edu/ ${ }^{\text {schoutens/ }}$ 